\title{
COVID19 Prevention \& Care; A Cancer Specific Guideline
}

Ali Motlagh, MD, MPH${ }^{1,2}$; Maisa Yamrali, MD*; Samira Azghandi, MD; Payam Azadeh, MD²; Mohammad Vaezi, MD'; Farzaneh Ashrafi,

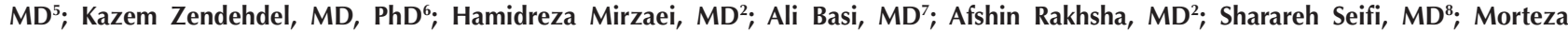
Tabatabaeefar, MD²; Ahmad Elahi, MD'; Pouneh Pirjani, MD'10; Leila Moadab Shoar, MD; Faranak Nadarkhani, MS ${ }^{11}$; Mostafa Khoshabi, MS $^{12}$; Massih Bahar, PhD ${ }^{13}$; Fatemeh Esfahani, MD ${ }^{8,14}$; Hossein Fudazi, MD ${ }^{15}$; Farhad Samiei, MD ${ }^{16,17}$; Borna Farazmand, MD ${ }^{18}$; Azin Ahmari, MD ${ }^{19}$; Mojtaba Vand Rajabpour, MD ${ }^{6}$; Ghasem Janbabaei, MD ${ }^{4,20}$; Alireza Raisi, MD ${ }^{21,22}$; Afshin Ostovar, MD, PhD ${ }^{1,23}$; Reza Malekzadeh $\mathrm{MD}^{1,24,25}$

${ }^{1}$ National Cancer Control Secretariat, Ministry of Health and Medical Education, Tehran, Iran ${ }^{2}$ Department of Radiation Oncology, Shaheed Beheshti Medical University, Tehran, Iran

${ }^{3}$ Department of Radiation Oncology, Iran University of Medical Sciences, Tehran, Iran

${ }^{4}$ Department of Hematology Oncology, Tehran University of Medical Sciences, Tehran, Iran

${ }^{5}$ Department of Hematology Oncology, Isfahan University of Medical Sciences, Isfahan, Iran

${ }^{6}$ Cancer Research Center, Cancer Institute of Iran, Tehran University of Medical Sciences, Tehran, Iran

'Department of Hematology Oncology, Iran University of Medical Sciences, Tehran, Iran

${ }^{8}$ Department of Hematology Oncology, Shaheed Beheshti Medical University, Tehran, Iran

${ }^{9}$ Department of Surgery, Alborz University of Medical Sciences, Karaj, Iran

${ }^{10} \mathrm{Ala}$ Cancer Prevention and Control Center (MACSA), Tehran, Iran

${ }^{11}$ Standard and Clinical Guidelines Department, Ministry of Health and Medical Education, Tehran, Iran

${ }^{12}$ Department of Geospatial Information System (GIS), Center of Excellence in GIS, K.N. Toosi Uni. of Technology, Tehran, Iran

${ }^{13}$ Behnam Daheshpour Charity Organization, Tehran, Iran

${ }^{14}$ Iranian Society of Medical Oncology and Hematology, Tehran, Iran

${ }^{15}$ Iranian Society of Radiation Oncology, Tehran, Iran

${ }^{16}$ Iranian Cancer Association, Tehran, Iran

${ }^{17}$ Department of Radiation Oncology, Tehran University of Medical Sciences, Tehran, Iran

${ }^{18}$ Department of Radiation Oncology, Mashhad University of Medical Sciences, Ghouchan, Iran

${ }^{19}$ Department of Radiation Oncology, Arak University of Medical Sciences, Arak, Iran

${ }^{20}$ Deputy of Curative Affairs, Ministry of Health and Medical Education, Tehran, Iran

${ }^{21}$ Department of Internal Medicine, Shiraz University of Medical Sciences, Shiraz, Iran

${ }^{22}$ Deputy of Public Health, Ministry of Health and Medical Education, Tehran, Iran

${ }^{23}$ Endocrinology and Metabolism Research Institute, Tehran University of Medical Sciences, Tehran, Iran

${ }^{24}$ Digestive Disease Research Institute, Tehran University of Medical Sciences, Tehran, Iran

${ }^{25}$ Deputy of Research and Technology, Ministry of Health and Medical Education, Tehran, Iran

\begin{abstract}
On March 11th 2020, the coronavirus outbreak was declared a pandemic by the WHO. One of the groups that is considered high risk in this pandemic are cancer patients as they are treated with a variety of immune system suppressor treatment modalities and this puts them in a great risk for infectious disease (including COVID-19). Therefore, cancer patients require higher level measures for preventing and treating infectious diseases. furthermore, cancer patients may bear additional risk due to the restriction of access to the routine diagnostic and therapeutic services during such epidemic. Since most of the attention of health systems is towards patients affected with COVID-19, the need for structured and unified approaches to COVID-19 prevention and care specific to cancer patients and cancer centers is felt more than ever. This article provides the recommendations and possible actions that should be considered by patients, their caregivers and families, physician, nurses, managers and staff of medical centers involved in cancer diagnosis and treatment. We pursued two major goals in our recommendations: first, limiting the exposure of cancer patients to medical environments and second, modifying the treatment modalities in a manner that reduces the probability of myelosuppression such as delaying elective diagnostic and therapeutic services, shortening the treatment course, or prolonging the interval between treatment courses.

Keywords: COVID-19, Cancer, Pandemic

Cite this article as: Motlagh A, Yamrali M, Azghandi S, Azadeh P, Vaezi M, Ashrafi F, et al. COVID19 prevention \& care; a cancer specific guideline. Arch Iran Med. 2020;23(4):255-264. doi: 10.34172/aim.2020.07.
\end{abstract}

Received: March 18, 2020, Accepted: March 21, 2020, ePublished: April 1, 2020 


\section{Introduction}

Coronavirus disease 2019 (COVID-19) is the new respiratory disease caused by the new corona virus. This virus is transmitted from person to person through respiratory secretions and contact (with surfaces or individuals) especially through Sneezing and coughing. ${ }^{1}$ Also, there is a possibility that it could be transmitted via aerosols. It is worth mentioning that some spread might be possible before people show any symptoms; but this is not thought to be the main way the virus spreads. ${ }^{2}$ This virus has a latency period of 1 to 14 days, mostly 3 to 7 days. ${ }^{1,3}$ Most common symptoms are fever, fatigue, dry cough and shortness of breath. A few patients may suffer from nasal congestion, nausea or vomiting and diarrhea. ${ }^{3}$

Currently we are in the middle of an ongoing global pandemic of COVID-19 and since cancer patients receive a variety of immunosuppressors, they are considered as a highrisk group for infectious diseases, including COVID-19, and require higher level measures for preventing and treating the infection. In addition, according to a report from China, cancer patients with COVID-19 disease had 3.5 times higher need for mechanical ventilation or ICU admission or death compared to patients without cancer. ${ }^{4}$

Iran was among the first countries that felt the necessity of having a national guideline designed for cancer patients and COVID-19, especially since few international guidelines were available. We developed a series of recommendations covering the continuum of care in this group of patients. The first group of recommendations is about the general precautions that should be taken by patients and their families, emphasizing the fact that preventive measures are of more importance in this population. The next series of recommendations focuses on the staff of medical centers (such as physicians, nurses, radiotherapy technicians, etc) and in addition to important protective measures for staff and patients, provides suggestions and recommendations on the appropriate steps that should be taken regarding the treatment course of these patients. We also prepared recommendations for managers of cancer centers describing their responsibilities in areas such as coordinated preventive measures, education, and regulations and arrangements needed to have an effective service delivery and reduce the burdens of this pandemic to the health system.

Our guideline is based on the current situation of our country in terms of equipment and facilities available, the structure of our health system and the conventional workflow that exists in our hospitals. Surely, these recommendations are not completely applicable for all countries struggling with this crisis, however, we believe that this guideline can be helpful in answering some important questions others may have and provide some solutions to current problems.

\section{Methods}

We conducted a nominal group discussion and involved a group of specialists in the National Cancer Control Secretariat of Iran. We used search engines such as PubMed and Google scholar and gathered and reviewed all the protocols and articles related to the subject of this guideline. Also, the latest protocols about COVID-19 prevention and treatment published by the Ministry of Health and Medical Education of Iran were reviewed as well. An initial draft of the guideline was prepared and it was shared with the advisory board who were scientists nominated from various universities, related scientific societies including Iranian Society of Radiation Oncology, Iranian Society of Medical Oncology and Hematology, Iranian Cancer Association, policy makers from deputies of public health, curative affairs, research and technology of Ministry of Health and Medical Education and a few cancer NGOs. Due to the risk of infection, the meetings were virtual and we used online systems to discuss the guideline. Each member of the advisory board received a draft of the prepared guideline and they wrote their comments. During this process, we repeated our search and found 4 articles ${ }^{5-8}$ with similar contents to our recommendations. These new articles were reviewed and some of their content was used in our article. Finally, the comments from the advisory board were gathered and incorporated. On March 2, 2020, the first edition of the guideline was prepared in Persian language. We issued the guideline for implementation to all medical universities, singed by the acting chair of National Cancer Control Committee, Ministry of Health and Medical Education. It is now in the process of implementation.

\section{Recommendations for Patients, Their Families and Caregivers}

General Recommendations

- Staying at home is the best way to protect yourselves from COVID-19.

- Have the least exposure possible to people, especially individuals with suspicious symptoms. Also, refrain from having any close contact with people (such as shaking hands, hugging or kissing).

- Pay special attention to symptoms like fever, cough, shortness of breath, chills, myalgia, headache, diarrhea, nausea, vomiting and runny nose, particularly within two weeks of exposure to an individual with any of these symptoms. ${ }^{3}$

- In case of sore throat, dry cough, chills with or without fever, or shortness of breath, contact your treating physician, the center you are being treated at or the centers providing COVID-19 care.

- Frequently wash your hands with soap and water for at least 20 seconds or use disinfectants containing at least 
$70 \%$ alcohol. ${ }^{9,10}$

- Wash your hands before having a meal, after using toilet, after sneezing or coughing and after having contact with suspicious surfaces. ${ }^{10}$

- When sneezing or coughing, cover your mouth and nose with a facial tissue or your elbow and immediately throw the tissue in a trash can. ${ }^{9}$

- Avoid touching your eyes or face. ${ }^{9}$

- Wear a face mask in circumstances such as being in a public place or medical center, or having respiratory symptoms like sneezing or coughing, or when giving care to a patient with fever and cough.?

- Regularly disinfect equipment that are frequently in contact with the hand, such as remote controls, door knobs, mobile phones, laptops or tablets. ${ }^{\text {? }}$

- Cook foods thoroughly, especially meat and eggs. ${ }^{11}$

- Be sure to regularly consume fresh fruits and vegetables that are properly washed. ${ }^{11}$

\section{In Medical Centers}

- Request your therapist to postpone or minimize diagnostic-therapeutic procedures to the extent that it does not harm your treatment, care and health.

- If it is necessary to be present in medical centers, minimize your presence time and avoid crowded waiting areas.

- Keep a distance of at least one meter from other people while waiting for a visit in the hospital or office lounge. ${ }^{12}$

- When attending medical centers, wear a face mask and preferably gloves and avoid touching your mouth, nose or eyes.

In Public Places

- Minimize your outdoor activities as much as possible. Avoid going to public or crowded places and keep a minimum distance of one meter. ${ }^{12}$

- If you have to go to public places, be sure to wear a face mask.

- Avoid touching public transportation surfaces and if so, wash and disinfect your hands the first chance you get.

- To press elevator buttons in public places use objects like napkins, cotton swabs or toothpicks and then, immediately throw them in the nearest trash can.

- In elevators, face the door not the people and avoid talking.

- Do not go to restaurants and if you have to, use disposable dishes and glasses.

- Use pedaled trash cans in public places.

- Wash your hands with soap and water or disinfectant solutions immediately after touching keyboards, computer mouse, phones, elevator buttons, ATMs, light switches, door bells of public places or houses, personnel fingerprint devices or stamps.?
At Home

- If you have been to hospitals, clinics or medical offices, immediately put your clothes in the washing machine on your return, take a shower and put on clean clothes.

- We recommend one person to be designated as the caregiver to the cancer patient: this person should pay more attention to sanitary recommendations and also have the least contact with other family members or outdoor environments.

- The caregiver should wear a face mask at all times of giving care to the cancer patient and should wash their hands before and after each care.

- The personal properties of the patient including personal hygiene supplies, utensils used for eating, and resting supplies such as pillows, bedding, and mattresses should be exclusive to the patient with cancer and separated from other family members.

- Make sure that the patient is kept separate from other family members in a private space and that he/she leaves this space as seldom as possible. Try to limit the visits of people who do not live in the house as much as possible. Given the higher risk of vascular thrombosis in cancer patients with ongoing treatment, limiting a patient to a room should not encourage a sedentary life and normal mobility should be emphasized.

- Limit your contact with other family members and minimize shared spaces in the house. Make sure that the shared spaces (such as kitchen or bathroom) are properly ventilated (for example, keep the windows open).

- We recommend that other family members have the least access possible to the patient's room.

- Make sure to disinfect all the surfaces the patient is in contact with (such as bedroom accessories and toilets) at least twice a day, using a tissue moisturized by a disinfectant solution.

- Check the body temperature of the cancer patient at least once a day: if it is higher than $37.3^{\circ} \mathrm{C}$ or if the patient has any symptom of dry cough, shortness of breath or feeling abnormally unwell, contact the treating physician or the center that has been providing the patient with its diagnostic-therapeutic services.

- Do not allow people with any symptoms of the common cold, even mildly runny nose or sneezing, to enter the patient's place of residence.

- Plan a balanced weekly diet for the patient and follow all the mentioned sanitary recommendations, especially regular hand washing while preparing the meal. ${ }^{11}$

- Make every effort to keep yourself and the patient calm and try to provide a stress-free environment at home. ${ }^{13}$

\section{Recommendations for Staff of Medical Centers}

- It is necessary for all the staff of cancer diagnosis and treatment centers to use masks (preferably N95) and gloves while working, including during disinfecting 
the surfaces.

- It is better to prepare the disinfectants in low volumes and for use in near future.

- Disinfect the surfaces (entrance and exit places, the surface of tables and chairs, door and window knobs, telephones) and other facilities at least once a day and preferably more often.

- It is necessary to disinfect the surfaces which the patients are in constant contact with (including the radiotherapy couch) several times a day and no less than twice.

- To disinfect the skin, it is necessary to use $70 \%$ alcohol $^{10}$ (3 portions of $96 \%$ alcohol and 1 portion of water).

- Disinfecting the environment, usual surfaces and the dishes can be done using $0.05 \%$ chlorine solutions (1 portion of 5\% bleaching agent and 99 portions of cold tap water) prepared in a plastic container with lid. ${ }^{14}$

- Disinfecting the environment and surfaces contaminated with patients' bodily fluids can be done using $0.5 \%$ chlorine solution (1 portion of $5 \%$ bleaching agent and 9 portions of cold tap water) prepared in a plastic container with lid. ${ }^{10}$

- First clean the dirty surfaces using detergents or soap and water, then wipe the surfaces using a clean tissue moisturized with disinfectant. Do not sweep. The remaining moisture on the surfaces must dry by itself and should not be wiped with another tissue. It is better to start at one end and finish at the other. Wash the tissue with separate water and bleaching solutions. Do this for all other surfaces.

- Only the patient has the permission to enter the inpatient or out-patient chemotherapy wards. Hand over all the items that patients are carrying to their companion.

- The chemotherapeutic drugs (and not their plastic bag) should be delivered to the nurse in a proper container before the patient enters the ward.

- When treating patients with radiotherapy, each patient should wear a disposable gown.

- It is necessary to wash your hands with antiseptic solutions or through away your gloves after each patient care and wear new ones.

\section{Recommendations for Physicians and Nurses}

- Two major goals are pursued in our recommendations; first, limiting the exposure of cancer patients to medical environments and second, modifying the treatment modalities in a manner that reduces the probability of myelosuppression such as delaying elective diagnostic and therapeutic services, shortening the treatment course, or prolonging the interval between treatment courses.

- In all circumstances and before making any decisions for each patient, it is necessary for the physician or the medical team to weigh the benefit from any diagnostictherapeutic intervention against the risks of possible infection with the COVID-19 virus, especially since cancer patients, as high-risk groups, have both higher probability of infection and higher probability of death from the COVID-19 virus.

- In all circumstances, it is necessary for the physician or the medical team to thoroughly explain the benefits and harms of any diagnostic-therapeutic intervention in the current situation to the patient (and with the patient's consent, to the family). The decision for starting or continuing the diagnostic-therapeutic interventions should be made via a shared decisionmaking process and individualized for each patient.

- We recommend postponing the screening services for asymptomatic patients. However, it is important to reschedule the patients' appointments to a time when the slowing down of epidemy is expected. ${ }^{5}$

- Taking into account all the measures mentioned above, curative surgeries should be planned and carried out as usual if the benefits are substantially higher than the potential loss due to COVID-19 infection or delay in treatment, with special attention to considerations regarding postoperative infections (Figure 1). ${ }^{5}$

- Regarding palliative surgeries, taking into account all the measures mentioned above, if a less risky alternative treatment exists which would not affect the patient's quality of life or survival, consider postponing the surgery (after taking into account the patient's considerations) (Figure 1).

- It is necessary to consider arrangements for postponing planned routine follow-up visits of patients with a history of cancer or requesting treatment response assessments (with imaging, etc.) if the patient has no clinical problems (Figure 1). ${ }^{6}$

- In circumstances where there are doubts about the benefits of radiotherapy or chemotherapy, based on the "level of evidence" or "level of recommendation", do not continue or start the treatment.

- Taking into account all the measures mentioned above, for patients who are being treated or are in the decision-making phase of treatment, the treating physician should talk with the patient and family about the benefits and harms of treatments (including chemotherapy and radiotherapy). Try as much as possible to postpone the palliative treatments or treatments such as bisphosphonate therapy that have less benefit in maintaining quality of life or increasing survival.

- Taking into account all the measures mentioned above, for patients who are being treated or are in the decision-making phase of treatment, the treating physician should talk with the patient and family about the benefits and harms of treatments (including chemotherapy and radiotherapy). Try to limit adjuvant 


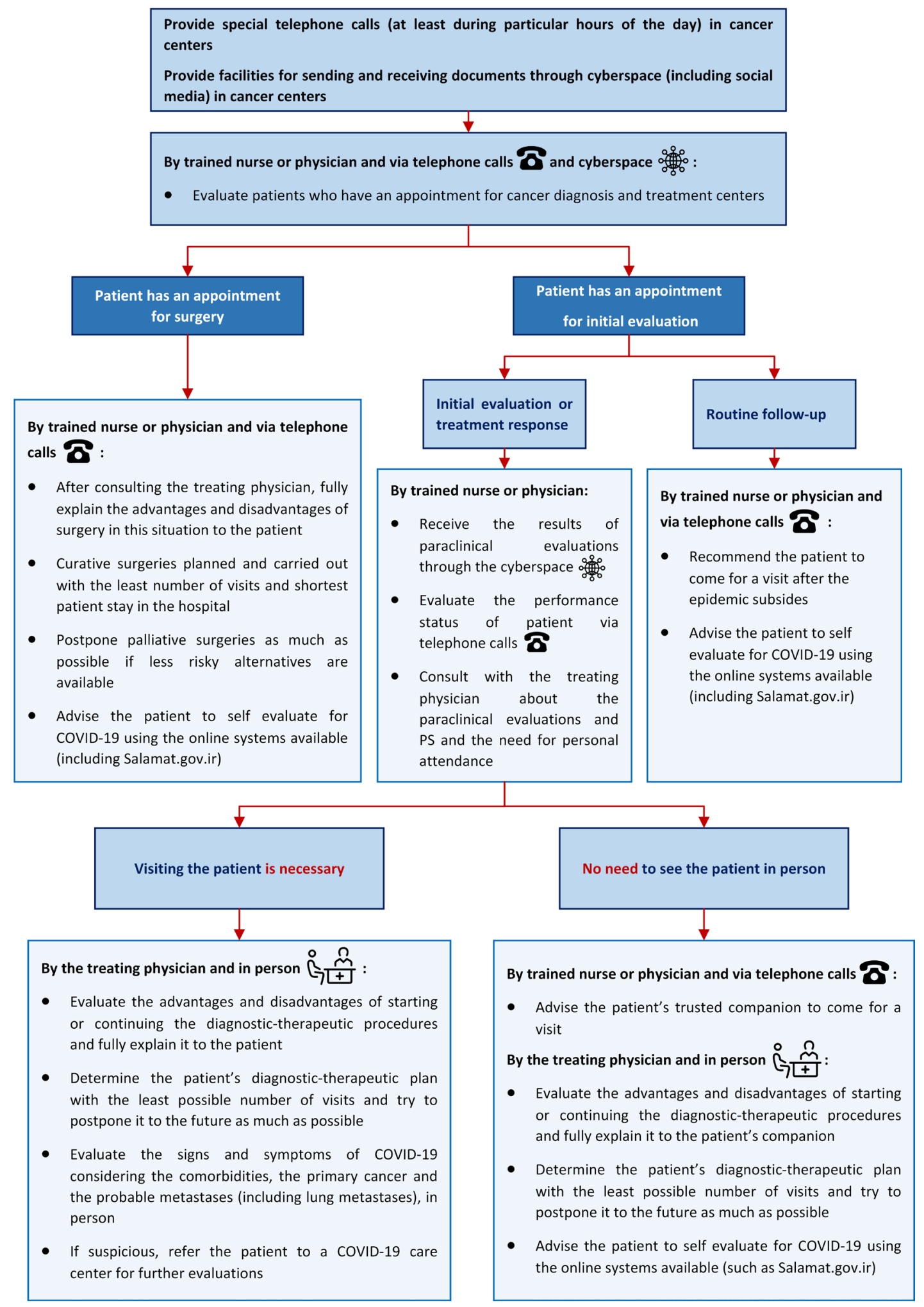

Figure 1. Cancer Patient Care During the COVID-19 Pandemic (Evaluations and Surgical Treatments).

or neo-adjuvant treatments to very beneficial ones and postpone others.

- Taking into account all the measures mentioned above, in situations where the benefits of chemotherapy outweigh the harms of probable infection with COVID-19 virus and alternative chemotherapy regimens can be chosen, consider the following alternatives (Figure 2):

- Prescribe outpatient chemotherapy regimens instead of inpatient ones in order to reduce the time spent by the patient in treatment centers. ${ }^{6}$

- Prescribe chemotherapy regimens with fewer number of courses instead of longer ones ${ }^{10}$ in order to reduce the probability of myelosuppression. 


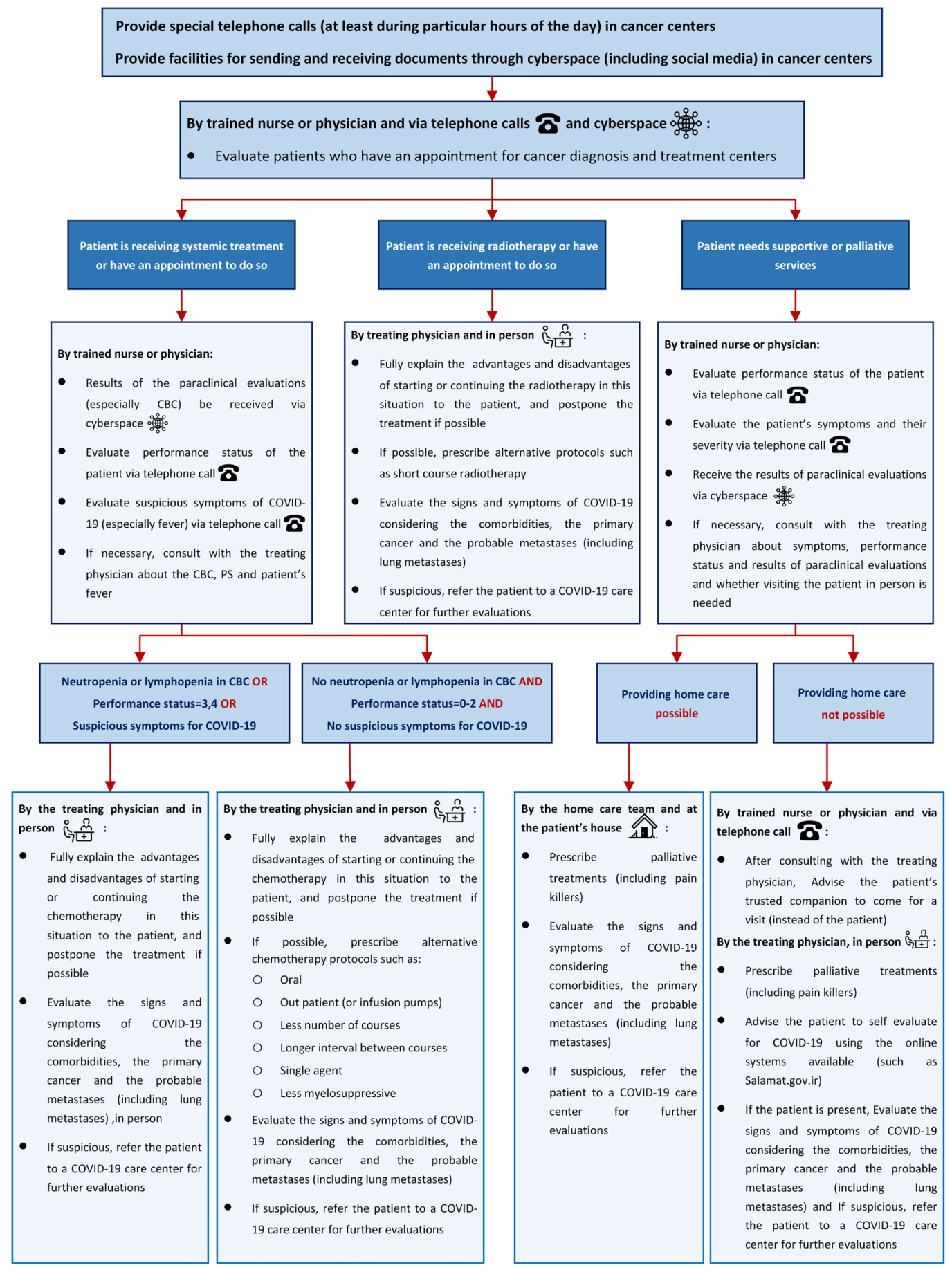

Figure 2. Cancer Patient Care During the COVID-19 Pandemic (Supportive and Palliative Care, Non-surgical Treatments).

- Prescribe chemotherapy regimens with longer intervals between courses instead of shorter ones (weekly) ${ }^{6}$ in order to reduce the time spent by the patient in treatment centers.

- Prescribe oral chemotherapy regimens instead of parenteral therapy in order to reduce the exposure of patients to treatment centers. ${ }^{6}$

- Use chemotherapy infusion pumps at home instead of inpatient chemotherapy regimens in order to reduce the exposure of patients to treatment centers. $^{6}$

- Prescribe less myelosuppressive chemotherapy regimens. ${ }^{6}$

- Prescribe single agent chemotherapy regimens instead of multi-drug ones in order to reduce the probability of myelosuppression.

- Taking into account all the measures mentioned above, when prescribing chemotherapy regimens with a high probability of myelosuppression, using prophylactic granulocyte-colony stimulating factor (G-CSF) therapy 
can be considered in some special circumstances taking into account 2 important issues:

- Thrombocytopenia is one of the important adverse effects of G-CSF therapy. Therefore, it is advisable to be extremely cautious when prescribing it with regimens that cause thrombocytopenia themselves (e.g. dacarbazine, carboplatin, fluorouracil, mitomycin).

- One of the other common complications of G-CSF therapy are flu like symptoms (generalized bone pain, fever or chills) and they can be mistaken with the COVID-19 symptoms, putting the patients and their families in great stress. Therefore, it is necessary to fully explain these complications to the patient in order to

- Taking into account all the measures mentioned above, in situations where the benefits of radiotherapy outweigh the harms of probable infection with COVID-19 virus and alternative protocols can be chosen (such as breast cancer, rectal cancer, prostate cancer or uncomplicated bone and brain metastases), prescribe short-course radiotherapy instead of longcourse radiotherapy (Figure 2). ${ }^{6,7}$

- It is necessary that all patients wear face masks when receiving their treatment (radiotherapy or chemotherapy). For radiotherapy of cranial or head and neck cancers, wearing a surgical mask under the thermoplastic mask is recommended. ${ }^{8}$

- Taking into account all the measures mentioned above, for prescribing chemotherapy drugs and evaluating the patients (including evaluating the performance status or laboratory results), limit the visiting sessions to one.
- If possible and in order to reduce the amount of commute, we recommend the medical team to use the capacities of cyberspace to communicate with the patients about their symptoms or tests and imaging evaluations. ${ }^{6}$

- Wearing a face mask is necessary (preferably N95) ${ }^{15}$ for the physicians and nurses working in cancer diagnosis and treatment centers.

- Nurses in outpatient and inpatient chemotherapy wards who are in close and inevitable contact with patients should preferably use proper gowns, N95 face masks and goggles. ${ }^{15}$

- In the case of bone marrow transplantation, the highest levels of protection for staff and patients should be considered.

- At each patient visit, pay close attention to suspicious symptoms and check the patient's body temperature.

- In patients with suspected symptoms of COVID-19 virus infection, especially fever and coughing, cancel chemotherapy and perform the necessary consultations as soon as possible (Figure 3).

- At each patient visit for chemotherapy and in case of suspecting COVID-19 virus infection, in addition to checking the count and percentage of neutrophils (ANC), pay close attention to the count of lymphocytes and probable lymphopenia (Figures 1, 2 and 3).

- Pay close attention to pulmonary imaging of patients with a history of cancer. Metastasis to lungs can sometimes be mistaken as pulmonary involvement by corona virus, especially plural effusions.

- When treating patients who are recovered from COVID-19, take a careful drug history and bear in

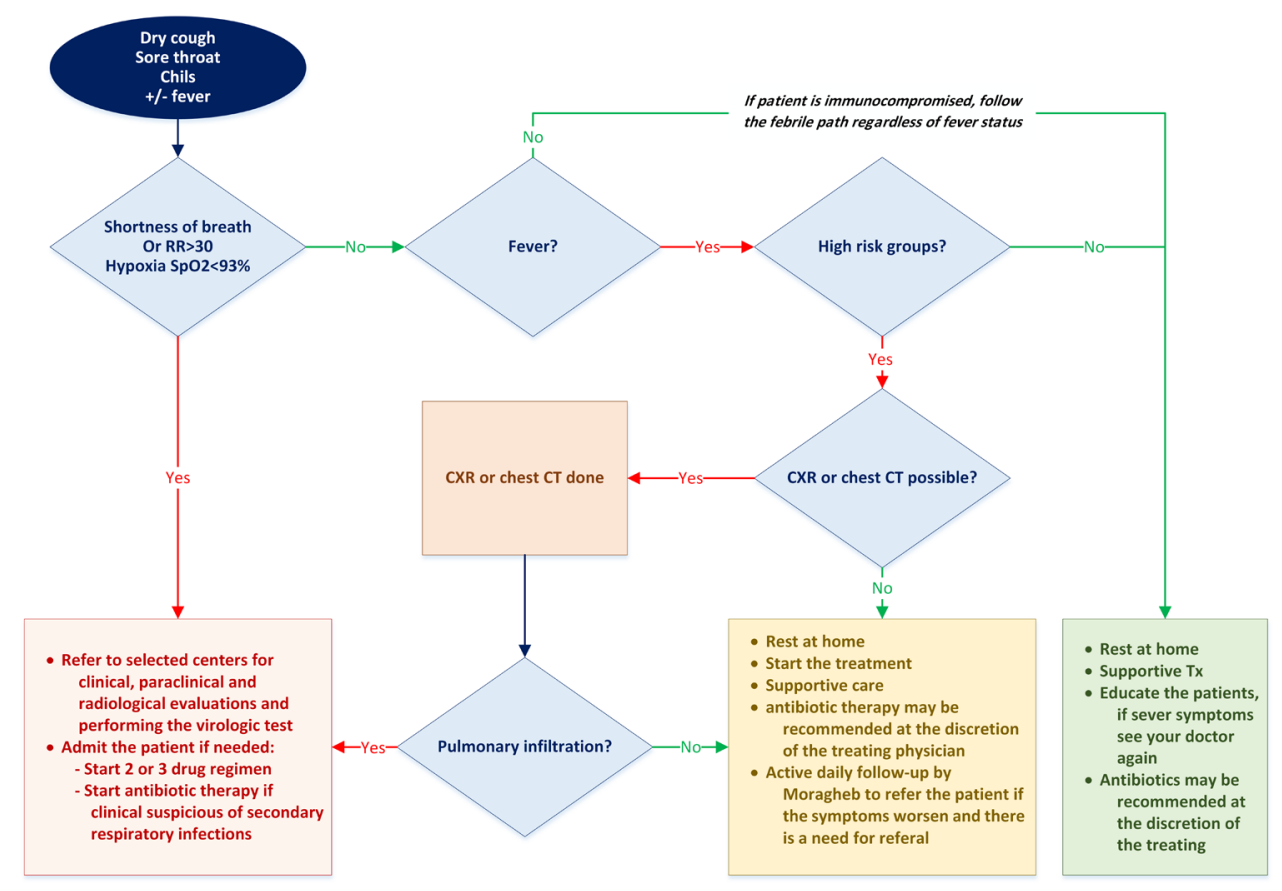

Figure 3. Flowchart for Diagnosis and Treatment of COVID-19 in Outpatient and Inpatient Settings in Iran. ${ }^{16}$ 
mind the possible cross reactions between antiviral drugs prescribed and the chemotherapy regimen you are planning to administer.

- If the physicians or nurses, who are involved in the treatment process of cancer patients, show any suspicious signs of COVID-19 virus infection, they should immediately exit the medical teams.

\section{Recommendations for Health System (Cancer Center) Managers}

- It is necessary to have weekly educational sessions for all the staff to update them about the newest information available and last changes in the guidelines especially with regard to cancer patients and COVID-19 (Table 1).

- Use teleconferencing facilities as much as possible, for example for holding the tumor boards, morning meeting or educational purposes.

- All the staff should be checked daily and before entering the ward for having a fever. Also, all staff should be educated about the symptoms of COVID-19 and in the case of having any related symptoms they should inform the manager of the center immediately and refer to COVID19 care center.

- Dividing the radiotherapy and chemotherapy wards into zones with different contamination levels is strongly recommended. ${ }^{8}$ The best policy would be to designate the radiotherapy room and the chemotherapy spaces special to each patient (chemotherapy bed and the utensils needed for injection) as the clean zone. Take all the measures necessary to make these spaces as corona virus free as possible. The station of radiotherapy technicians, the physics treatment planning room and the station of chemotherapy nurses are considered semi- clean spaces. The examination rooms and the waiting spaces are the contaminated zones. This zoning method helps to have a better decontamination policy in the mentioned wards.
- We recommend to arrange for a triage system in which all patients scheduled for treatment are screened for suspicious sings of COVID-19 via a telephone call by the responsible nurse before attending the treating center. Suspected patients should be visited in a separate room from other cancer patients and if the patient is still suspected for COVID-19, suspend the treatments and refer them to centers providing COVID-19 care.

- Since surgery is one of the main treatments for cancer, facilitate the conditions for performing curative surgeries. With regard to postponing palliative surgeries, take into account the physician's (or medical team's) opinion and the patient's condition.

- Make all the necessary arrangements to reduce the presence and gathering of patients or their companions in closed spaces of chemotherapy drugstores.

- Insurance companies should modify the process of confirming chemotherapy prescriptions to remote methods or temporarily postpone it.

- In circumstances in which chemotherapy is necessary, expand the service delivery time throughout the day (morning and evening shifts) in order to reduce contact between patients.

- It is necessary to reduce the waiting time of patients and their companions in medical centers by carefully adjusting radiotherapy and chemotherapy appointments.

- It is necessary to properly inform the patients who are planned to have routine follow-up visits to return at another convenient time when the epidemic subsides.

- Properly inform the patients in need of services to bring only one companion with them to the medical centers and preferably limited to underage or non-selfsufficient patients 5 .

- Inpatient visits by family and friends should be prohibited except in exceptional circumstances.

- Inform the patients and their companions to wear a proper face mask while in medical centers.

Table 1. Recommendations for Health System (Cancer Centers) Managers

\begin{tabular}{|c|c|c|c|}
\hline \multicolumn{2}{|l|}{ Education } & - & $\begin{array}{l}\text { Weekly educational sessions to update all the staff about the newest information and last changes in } \\
\text { guidelines including cancer patients and COVID-19 } \\
\text { Educating the staff about the symptoms of COVID-19 for daily self-evaluation } \\
\text { Providing educational pamphlets for cancer patients }\end{array}$ \\
\hline \multicolumn{2}{|c|}{$\begin{array}{l}\text { Utilizing the cyberspace and tele } \\
\text { facilities }\end{array}$} & & $\begin{array}{l}\text { Holding the morning meetings, educational classes and tumor boards using teleconferencing facilities } \\
\text { Creating a call center to answer the questions of patients and their families (via internet or telephone calls) } \\
\text { Planning a tele triage system for patients having an appointment in that center (via internet or telephone calls) }\end{array}$ \\
\hline \multirow[b]{2}{*}{$\begin{array}{l}\text { Necessary } \\
\text { regulations and } \\
\text { arrangements }\end{array}$} & $\begin{array}{l}\text { For personnel } \\
\text { and wards }\end{array}$ & - & $\begin{array}{l}\text { Checking the body temperature of staff daily and before entering the wards } \\
\text { Dividing the radiotherapy and chemotherapy wards into zones with different contamination levels (clean, } \\
\text { semi-clean, contaminated) } \\
\text { Providing home care services if possible }\end{array}$ \\
\hline & For patients & - & $\begin{array}{l}\text { Reducing the duration of patient stay in hospitals (expanding the service delivery time throughout the day, } \\
\text { adjusting the treatment appointments properly ...) } \\
\text { Limiting the number of patient companions } \\
\text { Prohibiting the inpatient visits } \\
\text { Wearing a face mask be mandatory for patients and their companions } \\
\text { Checking the body temperature of patients and companions at the entrance of wards }\end{array}$ \\
\hline
\end{tabular}


- Check the patients' and their companions' body temperature at the entrance; if they have fever, refer them to the emergency room or a center giving care to COVID-19 patients.

- If possible, provide the patients with sanitary packages containing facemasks, gloves and disinfectant solutions on arrival to use them at that instance.

- Educational pamphlets should be provided to all attending patients (Table 1).

- If proper facilities exist, create a call center with parttime or full-time answering capacity to patients and their families in order to reduce the number of patients turning to medical centers in person (Table 1, Figures 1 and 2).

- If the possibility of providing home care services exists, patients should receive medical care, especially supportive and palliative care, at home. Home care services will only include services authorized for home-based services and in accordance with previous guidelines issued by the Ministry of Health and Medical Education and it does not include chemotherapy services. In addition, before sending any home care team, it is important to ensure that they are healthy and that there are no suspicious signs of COVID-19 virus infection, and that appropriate masks, gloves and gowns are used (Figure 2).

\section{Recommendations for NGOs}

- In order to reduce face-to-face exposure, provide remote service delivery as much as possible.

- If possible, replace in-person services with at-home services.

- When providing at-home services, safety measures including wearing face masks and proper gowns and gloves are essential.

- Increase the awareness of cancer patients using educational pamphlets or video training programs through cyberspace.

\section{Implementation and Future Direction}

The guideline was prepared rapidly by acitve involvemnt of the specialists, governmental and non governmental organizations and medical univeristies. During the process of developing this guideline, a group from other insitiutes published some articles about cancer care during COVID-19 epidemic. ${ }^{5-8}$ However, this guideline is more comprelensive and prodided recommendations for patients, care givers and different groups who are involded in the manamgent of cancer patients. Since the overal knowledge and guidance about prevention and treament of COVID-19 is progressing rapidly, it is important to update this guideline regularly. In addition, the challenges on the implementation of this guideline will be discussed in the updated version.

\section{Authors' Contribution}

AM conceived of the presented idea and designed the basis of the manuscript and supervised the project. MY took the lead in writing the manuscript. All authors provided critical feedback and helped shape the research, analysis and manuscript. All authors read and approved the final manuscript.

\section{Conflict of Interest Disclosures}

None.

\section{Ethical Statement}

The authors declare that there is no conflict of interest regarding the publication of this article.

\section{Acknowledgements}

We wish to extend our special thanks to all the efforts made by NGOs, namely Mrs. Freshteh Salavati, Mrs. Arezou Dehghani, Mr. Amar Ghalenoie (National Network of Cancer charities and NGOs), Mrs. Neda Dadkhah (Mahak institute), Mrs. Leili Rezaei and Mrs. Fatemeh Khodsiani (Behnam Daheshpour charity).

\section{References}

1. Rothan HA, Byrareddy SN. The epidemiology and pathogenesis of coronavirus disease (COVID-19) outbreak. J Autoimmun. 2020 Feb 26:102433. doi: 10.1016/j.jaut.2020.102433.

2. "Coronavirus Disease 2019 (COVID-19), how it spreads". Centers for Disease Control and Prevention. March 4, 2020. Available from: https://www.cdc.gov/coronavirus/2019-ncov/ prepare/transmission.html.

3. "Symptoms of Novel Coronavirus (2019-nCoV)". US Centers for Disease Control and Prevention. March 20, 2020. Available from: https://www.cdc.gov/coronavirus/2019-ncov/ symptoms-testing/symptoms.html.

4. Liang W, Guan W, Chen R, Wang W, Li J, Xu K, et al. Cancer patients in SARS-CoV-2 infection: a nationwide analysis in China. Lancet Oncol 2020;21:335-7. doi: 10.1016/S14702045(20)30096-6.

5. Francesco C, Pettke A, Michele B, Fabio P, Helleday T. Managing COVID-19 in the oncology clinic and avoiding the distraction effect. Annals of oncology: official journal of the European Society for Medical Oncology. 2020 Mar 19. doi: 10.1016/j.annonc.2020.03.286.

6. Weinkove R, McQuilten Z, Adler J, Agar M, Blyth E, Cheng A, et al. Managing haematology and oncology patients during the COVID-19 pandemic: interim consensus guidance. Med J Aust. 2020;212(10):1.

7. Achard V, Tsoutsou P, Zilli T. Radiotherapy in the time of the Coronavirus pandemic: when less is better. Int J Radiat Oncol Biol Phys. 2020 Mar 19. doi: 10.1016/j.ijrobp.2020.03.008.

8. Wuhan CH, Zheng D, Liu Y, Hu D, Wei W, Han G. Advances in Radiation Oncology. Radiotherapy care during a major outbreak of COVID-19 in Wuhan. Available from: https:// preprod.astro.org/ASTRO/media/ASTRO/Daily\%20Practice/ PDFs/COVID-Han-et-al-(ADRO).pdf.

9. How to protect yourself from Novel Coronavirus (2019-nCoV). US Centers for Disease Control and Prevention. March 18, 2020. Available from: https://www.cdc.gov/coronavirus/2019ncov/prepare/prevention.html.

10. Water, sanitation, hygiene and waste management for COVID-19. Geneva: World Health Organization; 2020. Available from: https://www.who.int/publications-detail/ water-sanitation-hygiene-and-waste-management-forcovid-19.

11. Ministry of health and education, office of Community Nutrition Improvement, "Nutrition and preventing the new corona virus". 2020 Feb. Available from: http://health.behdasht. gov.ir/index.jsp?fkeyid $=\&$ siteid $=435 \&$ pageid $=58559 \&$ catid $=298 \& d v i$ ew $=1314$. 
12. COVID-19 advice for the public. Geneva: World Health Organization; 2020. Available from: https://www.who.int/ emergencies/diseases/novel-coronavirus-2019/advice-forpublic.

13. Ministry of health and education, office of Mental Health, "stress management and COVID-19". 2020 Feb. Available from: http://health.behdasht.gov.ir/index.jsp?fkeyid=\&siteid=435\&pa geid $=58559 \&$ catid $=298 \&$ dview $=1305$.

14. Ministry of health and education, center for communicable disease management, "environmental health measures to prevent new corona virus". 2020 Feb 22. Available from: http://health.behdasht.gov.ir/index.jsp? fkeyid $=\&$ siteid $=435 \&$ pageid $=5$ 8559 \& catid $=298 \&$ dview $=1315$.
15. Advice on the use of masks in the community, during home care and in healthcare settings in the context of the novel coronavirus (2019-nCoV) outbreak. Geneva: World Health Organization; 2020. Available from: https://www.who. int/publications-detail/advice-on-the-use-of-masks-in-thecommunity-during-home-care-and-in-healthcare-settings-inthe-context-of-the-novel-coronavirus-(2019-ncov)-outbreak

16. Ministry of health and education, "Flowchart for diagnosis and treatment of COVID-19 in outpatient and inpatient settings in Iran". 2020 Mar 13. Available from: http://medcare.behdasht.gov. ir/uploads/\%D\%86\%9D\%8B\%3D\%8AE\%D87\%9_\%D\%8B\%3D8\%9 \%8D85\%9_\%D\%81\%9D\%84\%9D\%88\%9DA\%86\%D\%8A\%7D\%8 B\%1D\%8AA.pdf. 